\title{
On the Relationship between the Wigner-Moyal and Bohm Approaches to Quantum Mechanics: A Step to a more General Theory?
}

\author{
B. J. Hiley* \\ TPRU, Birkbeck, University of London, Malet Street, \\ London WC1E 7HX. \\ Found.Phys.,40 (2009) 356-67.
}

\begin{abstract}
In this paper we show that the formalism used by Bohm in his approach to quantum mechanics is already contained in the earlier paper by Moyal which forms the basis for the Wigner-Moyal approach. This shows, contrary to the usual perception, that there is a deep relation between the two approaches. We suggest the relevance of this result to the more general problem of constructing a quantum geometry.
\end{abstract}

\section{Introduction}

It is a pleasure to contribute to this issue as Jeff Bub and I go back a long way. We were members of the Physics Department at Birkbeck College at the same time as David Bohm was appointed to a Chair in Theoretical Physics. I had just completed my $\mathrm{PhD}$ in condensed matter physics under the supervision of Cyril Domb and Michael Fisher and had been appointed to the lowly post of Assistant Lecturer, although who I was supposed to assist was never made clear. Jeff, fresh from Johannesburg, signed on for a $\mathrm{PhD}$ under Bohm's supervision. He was interested in the foundations of quantum mechanics and I wanted to move out of condensed matter physics to do something of a more fundamental nature. In a sense we were both embarking on the similar journey at the same time.

\footnotetext{
*E-mail address b.hiley@bbk.ac.uk.
} 
Jeff became interested in the Weiner-Segal approach [1] to quantum mechanics, a topic that became an important element of his thesis. He also engaged in a lively debate on the existence of hidden variables with Jauch and Piron [2]. While such debates have produced useful results, at that time I did not feel they would be very fruitful in taking us further in our quest for a deeper understanding of quantum phenomena.

During this period Roger Penrose was in the Mathematics Department at Birkbeck developing his ideas on twistors [3] and we used to hold weekly seminars ranging over many diverse topics in the foundations of physics, all challenging the conventional wisdom. During these seminars I was particularly fascinated by the notions of quantum space times that were beginning to be discussed at the time. I was particularly fascinated by John Wheeler's "sum over three geometries" ideas that he was using to quantise gravity. I remembering him lecturing in London at the time and he produced one of his inimitable drawings that showed a set of three geometries lined up on the shelves of a book case! In figure 1 I reproduce some of Wheeler's menagerie of three geometries [4] that made quite an impression on me.

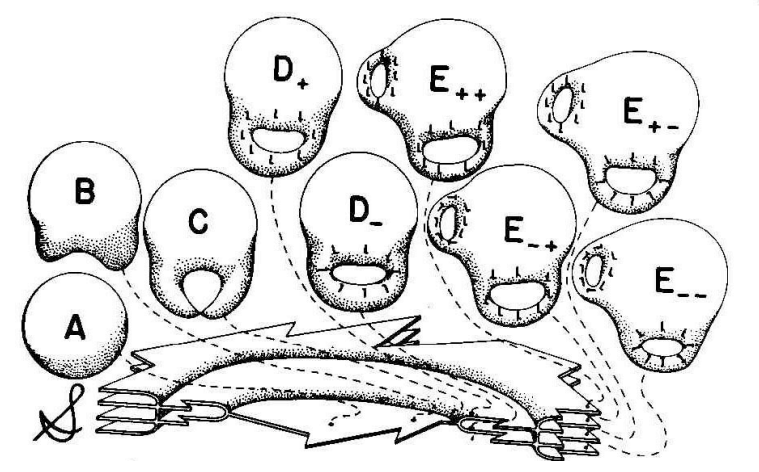

Figure 1: The multisheeted character of superspace.

It was the properties of the spinor that gave rise to the multi-sheeted character of the manifold. Here Wheeler had in mind the Pauli spinor. On the other hand, Penrose was generalising the spin structure by adding the twistor, a spin object that occurred in conformal geometry. Part of his twistor programme was to construct a quantum geometry in which the twistor variables would form a generalised phase space in which the consequences of curved space-time could be made manifest [5]. In presenting his ideas on the twistor, Penrose used a notation that was peppered with 
indices and I found it difficult to understand how it all fitted together. It eventually dawned on me that there was a beautiful general mathematical theory that lay behind these spin structures, namely the Clifford algebras where one could dispense with many of the indices, making life a little easier to see a rich structure emerging. These algebras formed a hierarchy of increasing dimension in which the Pauli spinor, the Dirac spinor and the twistor appeared at different levels in the hierarchy [6].

However all these algebras describe a classical geometry and it is not clear where the quantum aspects would come in. Wheeler did try to connect to the quantum domain by first starting with the classical Hamilton-Jacobi theory and then trying to find what he called a "Einstein-Schrödinger equation", as did Penrose within his twistor phase space, However both met with limited success [7]. Nevertheless I felt that there was something compelling about the whole approach.

One of the remarkable things about the sixties at Birkbeck was that, as far as I recall, we never discussed in any detail Bohm's original 1952 paper [8] that forms the basis of what later became known as "Bohmian mechanics" [9],[10]. Bohm himself was looking for a more fundamental structure of ideas upon which to base a new quantum theory. He was more interested in developing a process based philosophy in which new forms of order and structure would play a dominant role. It was, in fact, the beginning of what he eventually called the implicate order [11]. Here Bohm proposed that the mathematics best suited to describe order was the algebra and we explored some aspects of Clifford algebras hoping to find how from his general notion of order, space-time itself would arise [12]. This 'pre-space' order would form the basis of what people were calling quantum space-time [13].

All of this seemed a long way from the original ideas lying behind the '52 paper. This was why the Bohm model did not feature in our discussions. However when my attention was finally drawn to this paper, I suddenly realised that here was a possibility of finding clues to further Wheeler's ideas. The approach provided us with 'geodesics' and it also provided us with a quantum Hamilton-Jacobi equation. Could a detailed study of this model provide the clues necessary to find the elusive "Einstein-Schrödinger equation"? It was this question that motivated me to become involved in a detailed study of the full implications of the Bohm model. Some of the results of these investigations were presented in the book, "The Undivided Universe", that I co-authored with Bohm [14].

In this paper I want to report some new work that continues this line of investigation. At first sight it might appear that it has little direct relevance to the understanding of 'quantum space-times'. However what I have come 
to realise is that to supply the missing quantum aspects to the classical geometric structures of space-time we need to investigate quantum phase spaces. But surely, you protest, the construction of a phase space is the very thing that quantum mechanics denies? Seemingly not. Both the Bohm approach [14] and the Wigner-Moyal approach [23] introduce phase spaces and yet both produce exactly the same statistical predictions as standard quantum mechanics. There is an extensive literature on both topics yet very little is written on the relation between the two phase spaces constructed in each case. I will spell out the relationship in this paper and discuss the implications for the more general programme.

\section{Two phase space approaches?}

Over the years the original motivations for the work of Wigner [15], Moyal [16] and Bohm [8] seem to have been forgotten and their work is often presented as attempts to revert to a 'classical conception of nature', yet each paper contains clear warnings that their work does not imply a return to classical physics. In the case of Bohm, this point is, perhaps, brought out in more detail in his book "Chance and Causality in Modern Physics" [17].

Wigner [15] was interested in quantum corrections to the Boltzmann distribution used in thermodynamic equilibrium and involved many particles. However as he pointed out in the paper, it is possible to use the same formalism to account for the statistical properties of single quantum systems described by a pure state wave function. Even in this case we have a function given by

$$
F(x, p)=\frac{1}{\hbar} \int_{-\infty}^{\infty} \psi^{*}(x-y) e^{2 i p y / \hbar} \psi(x+y) d y
$$

and this can play the role of a 'probability distribution function' even though it takes negative values. Using it we can calculate the same expectation values as those calculated using the standard Hilbert space approach. We do this by simply integrating the operator equivalents over the position and momentum as one does in classical physics (see equation (8)).

Moyal's approach [16] starts by asking whether the standard formalism might not disguise what is essentially a generalised statistical theory, but one that is not necessarily based on a "crypto-deterministic" theory. He starts by taking a pair of non-commuting operators. (In this discussion we use the operators $\hat{p}$ and $\hat{x}$ with eigenvalues $p$ and $x$ to illustrate the method.) He then defines $M(\tau, \theta)$ to be the expectation value of the Heisenberg operator 
$e^{i(\tau \hat{p}+\theta \hat{x})}$. In statistics this object looks like the operator equivalent of the characteristic function of the distribution. In the state $\psi(x), M(\tau, \theta)$ takes the form

$$
M(\tau, \theta)=\int\left(\psi^{*}(x), e^{i(\tau \hat{p}+\theta \hat{x})} \psi(x)\right) d x
$$

where the RHS is the standard inner product. Then the Fourier transform of $M(\tau, \theta)$ is

$$
F(x, p)=\frac{1}{4 \pi^{2}} \iint M(\tau, \theta) e^{-i(\tau p+\theta x)} d \tau d \theta
$$

In turn this expression can then be written in the form [16]

$$
F(x, p)=\frac{1}{2 \pi} \int \psi^{*}(x-\hbar \tau / 2) e^{i \tau p} \psi(x+\hbar \tau / 2) d \tau
$$

which can be taken as a quasi probability distribution function. Apart from a trivial change of variable, this is identical to Wigner's original expression (1).

After showing how the characteristic function can be used to reproduce all quantum expectation values, Moyal goes on to discuss the implication of this approach. He notes that the distributions can take on negative values and concludes, therefore, that not all quantum states can be represented by true probability distributions. Therefore this cannot be regarded as a classical statistical theory. Nevertheless the existence of negative probabilities do not reveal themselves directly in any experiment and thus, from the practical point of view, we can use these functions to aide our calculations, a point of view that was originally suggested by Wigner [15] and later re-enforced by Feynman [18].

Bohm's approach [8] on the other hand was not motivated by an attempt to explain quantum mechanics in terms of a purely statistical theory. Rather he was concerned by the fact that there seemed to be no way in the conventional formalism to discuss what was happening between experiments. In other words there was no way of talking about a possible objective process that must be going on between measurements. The standard formalism implied that one could only talk about what happened when a measurement was made and there was no way to talk about what the particle may be doing between successive measurements. All we could do was to discuss the evolution of the wave function. For this reason the standard approach discourages any speculation as to what may be going on between measurements, believing we had reached the limits of the possibility of analysis in terms of particle trajectories etc. 
However in the WKB approximation, we can talk about what the particle could be doing between experiments. We can do this simply by neglecting terms of higher order than $\hbar$ in the resulting series expansion. But this process of approximation should not change the way we looked at the formalism as a whole. Yet here we have started with the quantum mechanical formalism with all its ambiguities and somehow "slipped over" into what is in essence, the ordinary classical ontology. How can simply throwing away terms in the WKB expansion enable one to go from ambiguity to what appears to be a deterministic theory? Why not keep all the terms and see what happens to the classical description? Will there be any contradictions? Will we learn anything new? It is not a question of forcing a classical view on the world, rather it is a question of seeing what is the exact difference between classical and quantum physics once both theories are reduced to the same mathematical language.

From the above remarks there seems on the surface to be no connection between the Wigner-Moyal and Bohm approaches. Both start from very different outlooks and what also appears to be a very different mathematics. Moyal's approach assumes an underlying stochastic process in a generalised phase space, the statistical distribution of which takes the form described by equation (3).

Bohm on the other hand starts from the Schrödinger equation itself and chooses to write the wave function in the polar form $\psi=R e^{i S / \hbar}$. The resulting real part of the equation then gives

$$
\frac{\partial S}{\partial t}+\frac{1}{2 m}(\nabla S)^{2}+V-\frac{\hbar^{2}}{2 m} \frac{\nabla^{2} R}{R}=0
$$

This is then treated as a modified Hamilton-Jacobi equation describing the evolution of a single quantum particle following one of the trajectories defined by the equation. It is this equation that we were referring to in the introduction when we discussed Wheeler's attempts to construct what he called the Einstein-Schrödinger equation. In our model we then simply assume that a particle can follow one of the ensemble of trajectories defined by the canonical relation

$$
p_{B}=\nabla S
$$

These then would correspond to the geodesics in the Wheeler approach [4]. Which particular trajectory a given particle travels along is unknown and uncontrollable since it is not possible to produce experimentally a single particle with a known simultaneous initial position and momentum. Thus we are unable to produce a single particle travelling down a chosen predetermined trajectory. All we are able to do is to produce a distribution 
of possible initial conditions and therefore possible trajectories. This is the contingent feature of the theory that gives rise to the statistics. The probability is then obtained by averaging over all initial positions assuming the distribution is consistent with the initial wave function. This final probability will always agree with the standard interpretation because the imaginary part of the Schrödinger equation gives the conservation of probability

$$
\frac{\partial \rho}{\partial t}+\nabla \cdot\left(\frac{\rho}{m} \nabla S\right)=0
$$

where $\rho(x)$ is the usual probability density $\rho(x)=\psi^{*}(x) \psi(x)$.

Again from these comments, it looks as if the two approaches are very different (see for example de Gosson [19]). Indeed, in certain circles, the Wigner-Moyal approach is regarded as a valid approach of exploring particle behaviour, whereas the Bohm approach is often dismissed as 'meaningless' [20]. However what I want to bring out in this paper is that the equations Bohm uses for his approach already appear in the appendix of Moyal's original 1949 paper [16]. This means that Bohm's formalism already sits within the Wigner-Moyal formalism. In this paper I want to show exactly how they are related.

\section{The Common Momentum.}

As we have already remarked, Moyal [16] starts by using the characteristic function defined in equation (2). He then constructs the expectation value of a given operator, $G(\hat{x}, \hat{p})$ by means of the following equation,

$$
\bar{G}=\iint G(x, p) F(x, p) d x d p
$$

where $G(x, p)$ is obtained from the operator form of $G$ by first using the Weyl procedure [21] viz.

$$
G(\hat{x}, \hat{p})=\iint \gamma(\theta, \tau) e^{i(\theta \hat{x}+\tau \hat{p})} d \theta d \tau
$$

and then forming

$$
G(x, p)=\iint \gamma(\theta, \tau) e^{i(\theta x+\tau p)} d \theta d \tau
$$

To make the connection with Bohm's formalism [8], we must follow Moyal's procedure of constructing the space-conditional moments of the momentum. These are written as $\overline{\bar{p}}$ and defined through

$$
\rho(x) \overline{\overline{p^{n}}}(x)=\int p^{n} F(x, p) d p
$$


Using the expression for $F(x, p)$ defined in equation (3) this can then be written in the form

$$
\rho(x) \overline{\bar{p}}^{n}(x)=\left(\frac{\hbar}{2 i}\right)\left[\left(\frac{\partial}{\partial x_{1}}-\frac{\partial}{\partial x_{2}}\right)^{n} \psi\left(x_{1}\right) \psi^{*}\left(x_{2}\right)\right]_{x_{1}=x_{2}=x}
$$

where $\rho(x)=\int F(x, p) d p=\psi^{*}(x) \psi(x)$. If we now write

$$
\psi(x)=\rho^{1 / 2}(x) e^{i S(x) / \hbar}
$$

we find for $n=1$

$$
\overline{\bar{p}}=\nabla S
$$

This is equation $(A 1.6)$ in the appendix of the paper by Moyal [16]. It is just the momentum defined in Bohm above through equation (5) .

\section{The Common Transport Equations}

\subsection{Transport of Probability}

To discuss transport equations we need to know how the quasi-probability distribution $F(x, p, t)$ evolves in time. The simplest way to present this is to follow Moyal and introduce a new product, the star product, defined by

$$
A(x, p) * B(x, p):=A(x, p) \exp \left[\frac{i \hbar}{2}\left(\frac{\overleftarrow{\partial}}{\partial x} \frac{\vec{\partial}}{\partial p}-\frac{\vec{\partial}}{\partial x} \frac{\overleftarrow{\partial}}{\partial p}\right)\right] B(x, p)
$$

Using this product we now introduce the Moyal bracket defined by

$$
\{A(x, p), B(x, p)\}_{M B}=\frac{A * B-B * A}{i \hbar}
$$

The star product sets up what is known as the deformed Poisson algebra, so called because if the Moyal bracket is expanded up to order $(\hbar)$, it becomes the classical Poisson bracket. Substituting equation (15) into equation (16) shows that the Moyal bracket can be written as

$$
\{A(x, p), B(x, p)\}_{M B}=A(x, p)\left(\frac{2}{\hbar}\right) \sin \left[\frac{\hbar}{2}\left(\frac{\overleftarrow{\partial}}{\partial x} \frac{\vec{\partial}}{\partial p}-\frac{\vec{\partial}}{\partial x} \frac{\overleftarrow{\partial}}{\partial p}\right)\right] B(x, p)
$$

Then the time development of the quasi-probability distribution can be written in the form

$$
\frac{\partial F(x, p)}{\partial t}+\{F(x, p), H(x, p)\}_{M B}=0
$$


In the limit to order $\hbar$, this equation becomes the classical Liouville equation.

If we write the Hamiltonian as $H(x, p)=p^{2} / 2 m+V(x)$ this equation can be written in the form [22]

$$
\frac{\partial F(x, p, t)}{\partial t}+\frac{p}{m} \cdot \nabla F(x, p, t)=\int J\left(x, p-p^{\prime}\right) F\left(p^{\prime}, x, t\right) d p^{\prime}
$$

where

$$
J\left(x, p-p^{\prime}\right)=-i \int[V(x-y / 2)-V(x+y / 2)] e^{i\left(p-p^{\prime}\right)} d y
$$

This can also be written in the form

$$
J(x, p)=-\frac{2}{\hbar} \frac{1}{(2 \pi \hbar)^{3}} \int V(x+y / 2) \sin \left(\frac{p y}{\hbar}\right) d y
$$

If we integrate equation (19) over $p$ we find the RHS of equation (20) vanishes leaving

$$
\frac{\partial \rho}{\partial t}+\nabla \cdot\left(\frac{\rho}{m} \nabla S\right)=0
$$

which is just the equation for the conservation of probability. This equation appears as equation (A4.2) in Moyal's paper [16] and is identical to the Bohm equation (6).

\subsection{Transport of $\overline{\bar{p}}$}

In order to find the transport equation for $\overline{\bar{p}}$, we must multiply equation (19) by $p_{k}$ to obtain

$$
\frac{\partial p_{k} F(x, p, t)}{\partial t}+\sum_{i} \frac{p_{k} p_{i}}{m} \frac{\partial F(x, p, t)}{\partial x_{i}}=\int p_{k} J\left(x, p-p^{\prime}\right) F\left(x, p^{\prime}, t\right) d p^{\prime}
$$

Then integrating over $p$ we find the RHS of equation (19) reduces to $-\rho \partial V / \partial x_{k}$ so that the equation becomes

$$
\frac{\partial\left(\rho \overline{\overline{p_{k}}}\right)}{\partial t}+\frac{1}{m} \sum_{i} \frac{\partial}{\partial x_{i}}\left(\rho \overline{\overline{p_{i} p_{k}}}\right)=-\rho \frac{\partial V}{\partial x_{k}}
$$

We can also show that the dispersion in momentum becomes

$$
\frac{1}{m} \sum_{i} \frac{\partial}{\partial x_{i}}\left[\left(\rho \overline{\overline{p_{i} p_{k}}}\right)-\left(\rho \overline{\overline{p_{i}}} \cdot \overline{\overline{p_{k}}}\right)\right]=-\frac{\hbar^{2}}{4 m} \sum_{i} \frac{\partial}{\partial x_{i}}\left[\rho \frac{\partial^{2} \ln \rho}{\partial x_{i} \partial x_{k}}\right]
$$


We can now use this result in equation (23) so that it can be written in the form

$$
\frac{\partial\left(\rho \overline{\overline{p_{k}}}\right)}{\partial t}+\frac{1}{m} \sum_{i} \frac{\partial\left(\rho \overline{\overline{p_{i}}} \cdot \overline{\overline{p_{k}}}\right)}{\partial x_{k}}=-\rho \frac{\partial V}{\partial x_{k}}-\frac{\hbar^{2}}{4 m} \sum_{i} \frac{\partial}{\partial x_{i}}\left[\rho \frac{\partial^{2} \ln \rho}{\partial x_{i} \partial x_{k}}\right]
$$

Differentiating the first term in equation (25), using equation (14) we find

$$
\rho \frac{\partial}{\partial x_{k}}\left[\frac{\partial S}{\partial t}+\frac{1}{2 m} \sum_{i}\left(\frac{\partial S}{\partial x_{i}}\right)^{2}+V\right]=\frac{\hbar^{2}}{4 m} \sum_{i} \frac{\partial}{\partial x_{i}}\left[\rho \frac{\partial^{2} \ln \rho}{\partial x_{i} \partial x_{k}}\right]
$$

In order to bring the RHS of equation (26) into recognisable form let us write $\rho=R^{2}$. Then it is straight forward to show

$$
\frac{1}{4 m} \sum_{i} \frac{\partial}{\partial x_{i}}\left[\rho \frac{\partial^{2} \ln \rho}{\partial x_{i} \partial x_{k}}\right]=\frac{1}{2 m} \rho \frac{\partial}{\partial x_{k}} \sum_{i}\left[\frac{\partial^{2} R}{\partial x_{i}^{2}} / R\right]
$$

so that equation (25) becomes

$$
\rho \frac{\partial}{\partial x_{k}}\left[\frac{\partial S}{\partial t}+\frac{1}{2 m}(\nabla S)^{2}+V-\frac{\hbar^{2}}{2 m} \nabla^{2} R / R\right]=0
$$

Thus we immediately see the connection with the Bohm formalism as expressed through equation (4). Equation (28) is essentially equation (A 4.4) in Moyal's paper [16]. His equation is derived for a charged particle moving in an electromagnetic field using the more general Hamiltonian $H\left(p_{i}, x_{i}\right)=\frac{1}{2 m} \sum_{i}\left(p_{i}-\frac{e}{c} A_{i}\right)^{2}+V\left(x_{i}\right)$ and reduces to equation (28) in the absence of the vector potential. Thus the three essential equations that form the basis of the Bohm interpretation already appear in the appendix of Moyal's classic paper.

In passing it should be noted that by combining equations (24) and (27) we have

$$
\overline{\overline{p_{i} p_{k}}}-\overline{\overline{p_{i}}} \cdot \overline{\overline{p_{k}}}=\frac{\hbar^{2}}{2} \frac{\partial}{\partial x_{k}}\left[\frac{\partial^{2} R}{\partial x_{i}^{2}} / R\right]
$$

Thus we see that, mathematically, the quantum potential arises from difference between the mean of the square of the momentum and the mean momentum squared. All this means that the dispersion in the momentum for a single particle in quantum mechanics can be nonzero. For the single 
particle in classical physics the momentum is always dispersion free. Equation (29) shows that this difference can be written as the gradient of a quantity that has become known as the quantum potential.

The connection with the quantum potential is made even clearer when one realises that the LHS of equation (25) is the total derivative of mean momentum $\overline{\bar{p}}$, so that using equation (27) in equation (25) we find

$$
\frac{d \overline{\bar{v}}}{d t}=-\nabla[V+Q]
$$

where $Q$ is the quantum potential $Q=-\frac{\hbar^{2}}{2 m} \nabla^{2} R / R$. Equation (30) explains the origin of the name.

\section{Conclusions.}

What we have brought out in this paper is the mathematical relationship between the formalisms used by Moyal and by Bohm. Both introduce the same momentum $P_{B}=\overline{\bar{p}}$ and both produce the same quantum HamiltonJacobi equation (2). Although this relationship has already been pointed out by Takabayasi [22], it seems to have been forgotten in the present debate concerning the interpretations of the quantum formalism. Take for example the excellent review article by Carruthers and Zachariasen [23] which deals specifically with approaches based on quantum mechanical phase-space distributions. The original Wigner-Moyal work and its subsequent development is discussed in great detail, but the Bohm approach does not even get a mention. Yet the trajectories that play such a prominent role in the Bohm approach are none other than the stream lines of the mean momentum field $\overline{\overline{p(x, t)}}$ of the Moyal approach.

The reluctance to see the Bohm approach as an alternative representation of the quantum formalism has always puzzled me [20]. I have always seen it as a way of gaining a different insight into quantum phenomena. The reluctance to consider it seriously appears to come from the firm belief that the Heisenberg uncertainty principle 'forbids' us to attribute a simultaneous precise position and momentum to an individual quantum particle. However such a strong assertion cannot be justified by appealing to the uncertainty principle alone. What the uncertainty principle states is that it is not possible to measure simultaneously the precise position and momentum of such a particle, a feature that is built into the Bohm approach. Indeed that the Heisenberg uncertainty principle is about measurement has been backed up by many detailed analysis of experiments, both actual and hypothetical, 
that attempt to perform such a measurement. All fail, so this principle is in no doubt but in asserting this fact but one should note that it is all to do with measurement.

What can we conclude from this fact? It seems to me we can draw one of two conclusions. Either we can assume the principle implies that a quantum particle cannot possibly have a precise position and momentum. Alternatively we can simply assume that a quantum particle can have a precise position and momentum but we cannot measure and hence know the values of these variables simultaneously. Experiment cannot help to decide between these two possibilities because there is no actual experiment that will do it for us. In other words there is no experiment to directly show that a quantum particle cannot have precise values for these two variables. Equally there is no experiment to directly show that a quantum particle can have simultaneous position and momentum. Thus both the standard and phase-space approaches depend crucially on which assumption is adopted at this point.

Since the original assumption used by the Bohm approach cannot be tested directly, there has always been the hope that there may be some indirect experimental way to distinguish this approach from the standard approach. But how can there be any difference? Both the standard and phase-space approaches use exactly the same mathematical structure.

The Wigner-Moyal approach derives all its results starting from the twopoint density matrix equation. On the other hand the Bohm approach derives its results directly from the Schrödinger equation. Neither add any new mathematical content. Both of these approaches are designed to give exactly the same statistical results as the standard formalism therefore there cannot be any measurable difference. Therein lies the weakness of proposing these approaches as 'new' theories; they are not new theories. They are simply different ways of looking at the same formalism.

The question then is why should we explore these different approaches? They predict no new results that could not be deduced from the standard approach so why bother? One answer has already been outlined in the introduction. Once we have a different way of looking at the quantum formalism, we begin to see quantum phenomena in a different perspective so that we open up new possibilities. The fact that we can use the concept of a trajectory for a quantum particle suggests that in attempting to find a quantum theory of gravity, we do not have to construct a Hilbert space but can try to see how the geodesics of the classical theory may be modified to produce quantum behaviour. Of course I am not saying that, therefore, we have a quantum theory of gravity, far from it, but at least we have the 
possibility of using a similar mathematical language to discuss both topics.

Of course there is a more direct answer to the 'why bother' question. The standard approach giving rise to the well known apparently inexplicable paradoxes, particle behaviour in two-slit interference experiments, schizophrenic cats, delayed choice paradox, quantum erasure and the measurement problem, which lead to so many conceptual difficulties. The measurement problem in particular consumes an immense amount of intellectual energy and has generated a vast literature seemingly without and end in sight. The Bohm approach has none of these difficulties.

For me the discussion of the measurement problem in the standard approach always hints at a degree of contradiction. The original assumption in denying that a quantum particle has a simultaneous position and momentum was supported by what I can only describe as the 'feeling' that we should not be using properties that cannot be directly measured, fitting in with the prevailing mood of positivism at the time the standard interpretation was being established. But this assumption seems to have been abandoned when one decides to look for ways to "collapse the wave function". It is generally assumed that the wave function cannot be measured directly, yet in this whole discussion, we are implicitly treating it as something that is physical or "real" that has to "collapse". It is assumed to be part of the physical process even though it cannot be measured. Contrast that with the strong resistance to attribute simultaneous values of position and momentum to a particle.

All of this is not to imply that the phase-space approaches supply us with all the answers. The phase space that is constructed is not a classical space and on further investigation shows some interesting deeper structure. For example, as we have already remarked above, equation (19) can be obtained directly from a two-point density matrix $\rho=\psi^{*}(x) \psi\left(x^{\prime}\right)$. Changing variables to $X=\left(x+x^{\prime}\right) / 2$ and $y=x-x^{\prime}$, we find

$$
\rho(X-\hbar y / 2, X+\hbar y / 2)=\int F(X, P) e^{-i y P / \hbar} d P
$$

Thus the $(X, P)$ used in the Wigner distribution are the mean values of some form of cell structure in phase space, which hints at a deeper underlying topological structure [26]. de Gosson [27] also discusses these cell structures, calling them "quantum blobs". He shows that they are related to the topological notion of capacity, a notion that arises naturally in symplectic geometry. It is also interesting to note that $F(X, P)$ turns out to be identical to the ambiguity function used extensively in radar theory [24] [25] suggesting that we are looking at the average behaviour of some form of 
underlying cellular dynamics. All this suggests that some form of underlying small scale topology which could fluctuate, resembling the foam-like structure suggested by Wheeler in his proposals of what he calls "pre-geometry" $[4]$.

Some preliminary results of my own based on this type of topological ambiguity has been reported elsewhere [26]. However even this preliminary work suggests that a more radical approach based on non-commutative geometry [29] or more radically still on a form of non-commutative topology along the lines suggested by van Oystaeyen [28]. These ideas will be discuss elsewhere.

\section{Acknowledgements}

I should like to thank Professor Robert Rynasiewicz for inviting me to present some of these ideas at his conference "New Directions in the Foundations of Physics" held at the University of Maryland in April 2007.

\section{References}

[1] Bohm, D. and Bub, J., A Proposed Solution to the Measurement Problem in Quantum Mechanics by a Hidden Variable Theory, Rev. Mod, Phys., 38 (1966) 453-69.

[2] Bohm, D. and Bub, J., A Refutation of a Proof by Jauch and Piron that Hidden Variables can be Excluded in Quantum Mechanics, , Rev. Mod, Phys., 38 (1966) 470-5.

[3] Penrose, R., (1967), Twistor Algebra, J. Maths Phys., 8, 345-366.

[4] Wheeler, J. A., Superspace and the Nature of Quantum Geometrodynamics, in Battelle Rencontres, ed. DeWitt, C. M. and Wheeler, J. A., pp. 242-307, Benjamin, New York, 1968.

[5] Penrose, P., On the Nature of Quantum Geometry, in Magic without Magic, ed. Klauder, J. R., Freeman, San Francisco 1972.

[6] Francis, M. R. and Kosowsky, A., The Construction of Spinors in Geometric Algebra, math-ph/0403040.

[7] Penrose, R., The Road to Reality Vintage Books, London, 2004. 
[8] Bohm, D., A Suggested Interpretation of the Quantum Theory in Terms of Hidden Variables, I, Phys. Rev., 85, and II, 166-179; 85,180-193 (1952).

[9] Dürr, D., Goldstein, S. and Zanghi, N., Quantum Equilibrium and the Origin of Absolute Uncertainty, J. Stat. Phys., 67, 843-907, (1992).

[10] Dürr, D., Goldstein, S. and Zanghi, N., 1996, Bohmian Mechanics as the Foundation of Quantum Mechanics, in Bohmian Mechanics and Quantum Theory: an Appraisal, ed J. T. Cushing, A Fine and S. Goldstein, Boston Studies in the Philosophy of Science, 184, p. 26, Kluwer, Dordrecht.

[11] Bohm, D., Wholeness and the Implicate Order, Routledge, London, 1980 .

[12] Bohm, D. and Hiley, B. J., Twistors, Rev. Briasileira de Fisica, Volume Especial, Os 70 anos de Mario Schönberg, 1-26, (1984)

[13] Bohm, D., Time, the Implicate Order and Pre-Space, in Physics and the Ultimate Significance of Time, ed. D.R. Griffen, 172-6 and 177-208, SUNY Press, N.Y., (1986).

[14] Bohm, D. and Hiley, B. J., The Undivided Universe: an Ontological Interpretation of Quantum Theory, Routledge, London 1993.

[15] E. Wigner, On the Quantum Correction for Thermodynamic Equilibrium, Phys. Rev., 40, 749-59, (1932).

[16] J. E. Moyal, Quantum Mechanics as a Statistical, Theory,Proc. Cam. Phil. Soc., 45, 99-123. (1949).

[17] D. Bohm, Chance and Causality in Modern Physics, Routledge \& Kegan Paul, London (1957).

[18] R. P. Feynman, Negative Probability, in Quantum Implications, ed. B. J. Hiley and F. D. Peat, pp. 235-48, Routledge \& Kegan Paul, London, (1987)

[19] M. de Gosson, The Quantum Motion of Half-densities and the Derivation of Schrödinger's Equation, J. Phys, A: Math. Gen., 31, 4239-47, (1998).

[20] H. D. Zeh, Why Bohm's Quantum Theory?, Quant-ph/9812059. 
[21] H. Weyl, The Theory of Groups and Quantum Mechanics, p. 274, Dover, London, (1931).

[22] T. Takabayasi, The Formulation of Quantum Mechanics in terms of Ensemble in Phase Space, Prog. Theor. Phys., 11, 341-74, (1954)

[23] P. Carruthers and F. Zachariasen, Quantum Collision Theory with Phase-space Distributions, Rev. Mod. Phys., 55, 245-85, (1983).

[24] W. J. Schempp, Harmonic Analysis on the Heisenberg Nilpotent Lie Group with Applications to Signal Theory Pitman Research Notes in Mathematics Series, 147, Longman Scientific \& Technical, 1986.

[25] W. Moran and J. H. Manton, in Computational Noncommutative Algebra and Applications, ed. J. S. Byrnes, pp. 339-62, NATO Science Series, Kluwer Academic, (2004).

[26] B. J. Hiley, Towards a Dynamics of Moments: the Role of Algebraic Deformation and Inequivalent Vacuum States, in Correlations, ed. K. G. Bowden, Proc. ANPA 23, 104-134, (2001).

[27] M. de Gosson, Phys. Lett. A 330, 161-167, (2004).

[28] F. van Oystaeyen, Virtual Topology and Functor Geometry To be published 2006.

[29] B. J. Hiley, Phase Space Descriptions of Quantum Phenomena, Proc. Int. Conf. Quantum Theory: Reconsideration of Foundations, 2, 26786, ed. Khrennikov, A., Växjö University Press, Växjö, Sweden, (2003). 